\title{
An Evaluation Approach for Analyzing Workflow Management Systems from a Value-based Perspective
}

\author{
Bela Mutschler, Johannes Bumiller \\ DaimlerChrysler Research \& Technology \\ P.O. Box 2360, 89013 Ulm, Germany \\ \{bela.mutschler;johannes.bumiller\}@daimlerchrysler.com
}

\author{
Manfred Reichert \\ University of Twente \\ Information Systems Group \\ m.u.reichert@utwente.nl
}

\begin{abstract}
Workflow management systems (WfMS) have become a widely accepted software technology, which enables the effective management, execution, and monitoring of computerized processes. Though the introduction of WfMS results in high costs, corresponding investments are often justified solely by referring to assumed benefits such as improved business process performance. In fact, there exists no framework to systematically evaluate WfMS from a value-based perspective. One major reason for this drawback is that research about the economic impact of WfMS has to deal with many challenges like the potential identification of impact factors, the handling of dependencies among these impact factors, the collection of real-world data, and the quantification of costs and intangible benefits. This paper presents an evaluation methodology to tackle these problems, which is based upon modeling and simulation.
\end{abstract}

\section{Introduction}

Workflow management systems (WfMS) have become a widely accepted software technology in practice [5]. Very often, WfMS are embedded in middleware tools or in process-oriented applications. In particular, WfMS enable the effective management, execution, and monitoring of computerized processes.

Technical issues related to WfMS have been intensively investigated in literature [22]. By contrast, what has been neglected so far is the systematic analysis of costs and benefits related to the introduction of WfMS. In fact, projects implying the use of WfMS are usually justified by referring to technical feasibility and assumed benefits (e.g., improved business process performance $[14,18])$.

Picking up this issue, this paper presents an evaluation methodology to analyze the introduction of a WfMS from a value-based perspective. Impact factors and their dependencies are considered as "economic systems" whose structure and behavior are modeled and simulated in order to acquire knowledge about the economic impact of WfMS (e.g., through "what-if" analyses). Section 2 describes our evaluation methodology. Section 3 discusses related work. Finally, the paper concludes with a summary and an outlook in Section 4. The work presented in this paper is part of the EcoPOST project [1].

\section{Modeling and Simulating the Economics of Workflow Management Systems}

This section introduces our evaluation methodology to analyze WfMS from a value-based perspective. In particular, our approach is based on the design of systems of WfMS-specific evaluation variables. The structure and behavior of such systems are modeled and simulated to make economic effects transparent.

To build our evaluation models, we use System Dynamics $[9,15,19]$ as modeling formalism (instead of developing a new one). In particular, System Dynamics is well-suited for our research. Suitability in our context means to increase awareness regarding the economic impact resulting from the introduction of WfMS. Thus, System Dynamics is suitable as it can be considered as a conscious-raising tool [10]. In particular, System Dynamics enables us to systematically analyze economic-driven evaluation variables as well as their dependencies.

There are other modeling and simulation approaches that could be used as well. In particular, Bayesian Networks provide an interesting alternative. A Bayesian Network is a graphical description of probability distributions that permits probability propagation, i.e., the graph is used to calculate probabilities. A Bayesian Network is represented as a directed acyclic graph in which nodes reproduce vari- 
ables and edges indicate causal dependencies between pairs of variables. However, despite the structures of Bayesian Networks and System Dynamics seem to be similar, there is a fundamental difference between the meaning and applicability of both approaches. In particular, Bayesian Networks consider uncertainty, i.e., they allow for the determination of the probability of specific events. By contrast, System Dynamics does not model uncertainty. Its goal is to determine the impact of certain hypotheses. Dealing with uncertainty in our context, it becomes clear that we can identify the cost and impact factors that determine WfMS economics. We also know, for instance, that certain costs will occur (e.g., the costs related to the adaptation of process logic in the case of evolving processes). Thus, uncertainty has not to be handled and we decided to use System Dynamics. A detailed introduction to the formalism of System Dynamics can be found in [9, 19].

We analyze the introduction of WfMS from different viewpoints. In particular, we identify three perspectives ${ }^{1}$ :

1. The first evaluation perspective (EP1) covers the impact of WfMS on the efficiency and effectiveness of business processes, e.g., regarding the reduction of processing times due to the introduction of process automation technologies. Usually, quantifications based on processing times and process resources allocated constitute the evaluation baseline in this context.

2. The second evaluation perspective (EP2) analyzes the impact of WfMS on software development. This implies direct and indirect effects.

3. The third evaluation perspective (EP3) analyzes the impact of WfMS on software maintenance. As an example consider the need to adapt process-oriented information systems to changing business processes.

Figure 1 summarizes the major methodical steps of our approach. Step 1 deals with the specification of evaluation scenarios (Section 2.1). Step 2 identifies those factors that influence the specified evaluation scenario (Section 2.2). Step 3 concerns the design of evaluation models using the formalism of System Dynamics (Section 2.3). Finally, Step 4 deals with the interpretation of our evaluation models through computer simulation (Section 2.4).

\subsection{Specifying an Evaluation Scenario}

The first step of our evaluation methodology concerns the "research problem" to be analyzed: "Only by knowing

\footnotetext{
${ }^{1}$ Other evaluation perspectives, e.g., to evaluate the technical maturity of a WfMS, can be introduced as well if required.
}

the questions to be answered, we can safely judge the pertinence of factors to include in or omit from the system formulation" [9].

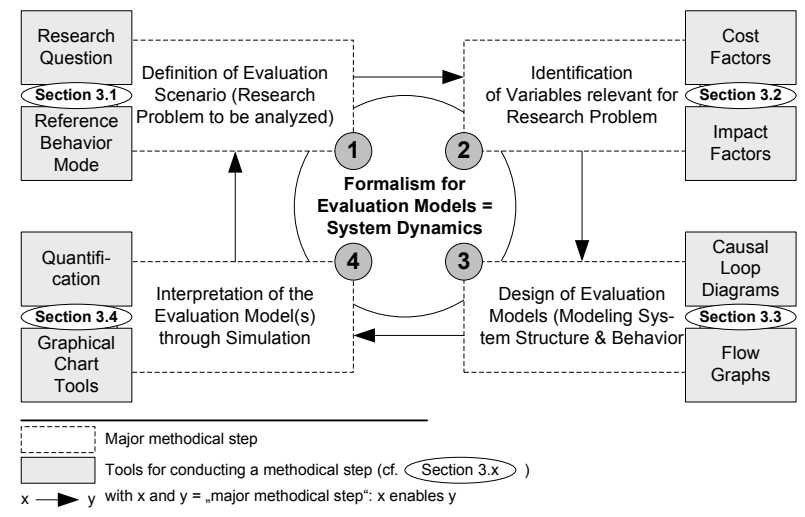

Figure 1. Methodology Overview.

Research problems are typically described using simple questions. For example, typical problems addressed in the context of our research are as follows: What costs are related to the introduction of a WfMS? What benefits? How does business process fragmentation influence the costs of introducing a WfMS? What economic effects are caused by explicitly describing process logic and by separating it from application code? How does "emotional resistance" of end users influence the costs of introducing a WfMS?

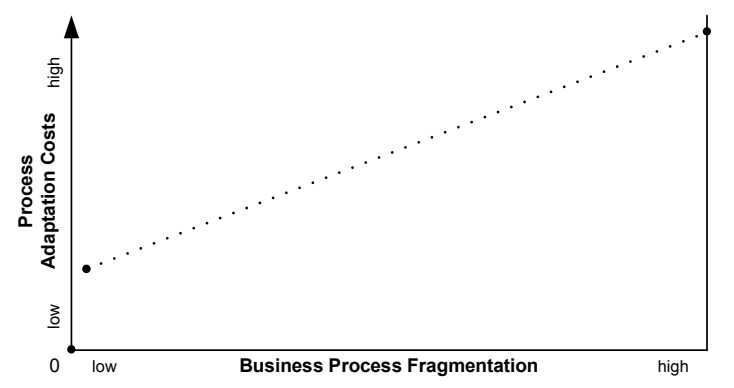

Figure 2. Specifying an Assumption.

To further enhance clarity, it is usual to specify an expected answer for a research problem by means of a graphical diagram. Such a diagram illustrates the assumed course of one or more model variables along the time axis. As an example consider the graphical diagram shown in Figure 2 . The diagram shows the assumed costs related to business process fragmentation. Here the logic of a particular business process is scattered over several process-oriented applications (e.g., legacy systems and off the shelf components). Changing the business process (i.e., its logic) may therefore necessitate the adaptation of all applications that 
deal with the changed process part. Typically, a high number of process fragments imply the need to adopt many applications when process changes occur. Consequently, the costs of process adaptation increase.

\subsection{Cost and Impact Factors}

The second step of our evaluation methodology deals with the identification of relevant evaluation variables.

Basically, our approach is cost-driven, i.e., our basic measure and subject to evaluation are costs (e.g., costs related to the introduction of a WfMS). Direct cost factors (cf. Fig. 3) are related to the WfMS itself. They include, for example, software licenses or project resources. Furthermore, we must also consider costs indirectly caused by the introduction and operational use of WfMS. As an example consider the efforts caused by the need to analyze and redesign the business processes to be automated. To identify both direct and indirect cost factors, we use existing methods such as total cost of ownership.

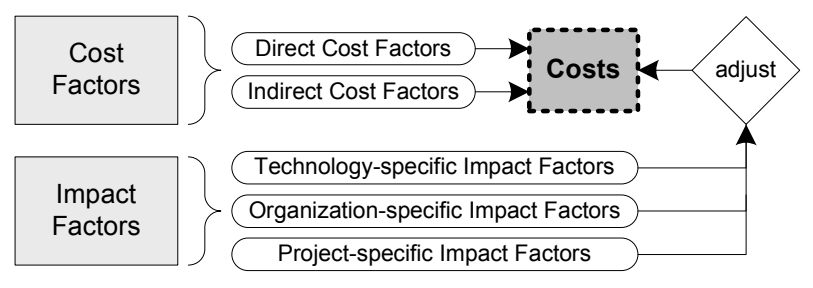

Figure 3. Cost and Impact Factors.

Besides, there are many intangible impact factors that additionally bias the costs of workflow technology. Often, these impact factors are difficult to quantify. In particular, we distinguish between three kinds of impact factors dependent on the level of their effectiveness:

- Technology-specific impact factors deal with technical capabilities of a WfMS. Examples include the degree of flexibility provided by the WfMS (e.g., regarding the support of dynamic changes) or the scalability offered.

- Organization-specific impact factors deal with organizational issues that bias the economics of a WfMS. As one example consider knowledge about organizationspecific processes. Another example concerns organizational barriers (e.g., between departments) that cause process interceptions.

- Project-specific impact factors deal with project factors that bias the economics of a WfMS. Examples concern the domain knowledge of IT professionals assigned to a project or the know-how of participating team members regarding the implementation of WfMS-based information systems.

A basic set of potential impact factors following this classification is given in Table 1. Many of them have been collected by Parkes $[16,17]$. Taking our practical experiences gained in the automotive domain, we can confirm most of these factors.

This set of impact factors represents a baseline for the design of evaluation models with our approach. However, it is subject to continuous review and extension. As always, it is sometimes difficult to clearly assign one impact factor to one of the aforementioned categories. Impact factors that could be also assigned to another category are therefore designated with the alternative categories (cf. Table 1).

\subsection{Model Formulation}

The third step of our evaluation methodology concerns the design of evaluation models. Evaluation models are "systems of variables". In the context of our research, variables are WfMS-specific cost and impact factors (see Section 2.2). In particular, we focus on the identification and modeling of causal dependencies among evaluation variables and analyze the dynamic behavior that is caused by these dependencies.

A causal dependency between two variables is depicted as an arrow (cf. Fig. 4). To illustrate the direction of causality, arrows can be labeled with a "+" or "." [21]. A label "+" indicates that the variable at the opposite end of the arrow tends to move in the same direction (in the case of a change). A label "-" indicates a reverse relationship. Arrows that are not labeled describe dependencies between variables with varying directions of causal influence. Consider the example shown in Figure 4. The model depicts the causal impact of cost and benefit variables on a WfMS's return of investment. Benefits increase the return of investment, whereas costs decrease this ratio.

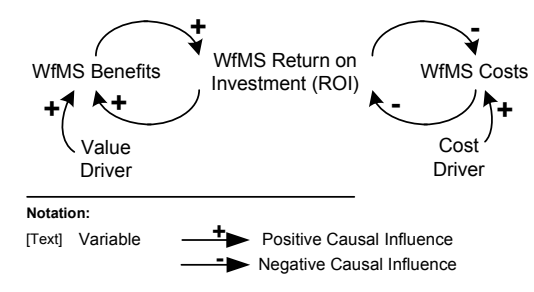

Figure 4. Modeling Causal Effects.

Dynamic system behavior is generated by chains of causes and effects within a system. Dynamic behavior implies that a system has different states over time. To be 


\begin{tabular}{|l|c|c|}
\hline I. Organization-specific Impact Factors \\
\hline 01: Process Transparency" & L & P \\
\hline 02: Knowledge about existing Business Processes" & L & P \\
\hline 03: Information about existing Business Processes" & L & P \\
\hline 04: Management Structure" & L & - \\
\hline 05: Management Commitment" & L & P \\
\hline 06: Need for Job Redesign" & L & P \\
\hline 07: End User Ownership" & L & - \\
\hline 08: Communication" & L & - \\
\hline 09: Changing Social Cue \& Interactions & L & P \\
\hline 10: Process Maturity & - & P \\
\hline 11: End User Fears \& Emotional Resistance" & - & P \\
\hline 12: Automation of Decision Making" & L & - \\
\hline
\end{tabular}

\begin{tabular}{|l|c|l|}
\hline II. Project-specific Impact Factors \\
\hline 01: Motivation for the Project & L & P \\
\hline 02: Ability to reengineer Processes' & L & P \\
\hline 03: Used Workflow Models' & L & - \\
\hline 04: Modeling Perspective & L & - \\
\hline 05: Access to required Skills' & L & P \\
\hline 06: Domain Knowledge' & L & P \\
\hline 07: Resource Redistribution & L & - \\
\hline 08: End User Participation' & L & P \\
\hline 09: Access to Information & L & - \\
\hline 10: Degree of potential WfMS Support'I' & - & P \\
\hline 11: Ability to use emerging Technology' & L & - \\
\hline 12: Business Process Fragmentation' & - & P \\
\hline 13: Process Evolution'l' & L & P \\
\hline 14: Planning of change' & L & P \\
\hline
\end{tabular}

\begin{tabular}{|c|c|c|}
\hline III. Technology-specific Impact Fact & & \\
\hline 01. Support for Groupwork Functionality & $\mathbf{L}$ & $\mathbf{P}$ \\
\hline 02: Required Technology Infrastructure" & $\mathbf{L}$ & - \\
\hline 03: Reuse of existing Technologies" & $\mathbf{L}$ & - \\
\hline 04: Supported Level of Change & $\mathbf{L}$ & $\mathbf{P}$ \\
\hline 05: Structural Modification & $\mathbf{L}$ & - \\
\hline 06: Number of IS to be Integrated" & $\mathbf{L}$ & $\mathbf{P}$ \\
\hline 07: Flexibility regarding Process Execution & - & $\mathbf{P}$ \\
\hline 08: Scalability of simultaneous Processes & - & $\mathbf{P}$ \\
\hline 09: Support of Standards and Norms" & - & $\mathbf{P}$ \\
\hline 10: Implementation of Process Logic & - & $\mathbf{P}$ \\
\hline 11: Support of Audit Trails & - & $\mathbf{P}$ \\
\hline
\end{tabular}

Table 1. Impact Factors determining the Economics of Workflow Management Systems.

able to trace these states, state variables continuously accumulate the results of actions in a system. A state is a snapshot of a system at a specific point in time. Subject to accumulation can either be tangible or intangible variables. State variables are graphically represented by a rectangle that comprises the name of the variable. State variables outside the analyzed system are called "sources" or "sinks". They are graphically represented by cloud-like symbols.

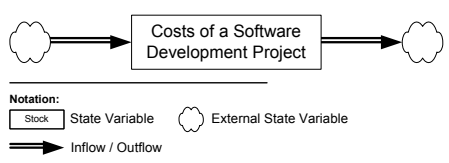

Figure 5. State Variables.

State variables, i.e., their accumulation, change only through inflows and outflows. Inflows increase and outflows decrease a state variable. Graphically, both inflows and outflows are depicted by twin-arrows pointing into or out of a state variable. As an example consider the state variable "Costs of a Software Development Project" (cf. Figure 5). The inflow increases the accumulated project costs. The outflow, in turn, decreases the project costs.

Inflows and outflows are controlled by rates. Thus, rates change accumulations of state variables. Graphically, rates are depicted by valves (cf. Fig. 6). The "Cost Rate", for example, controls the increase of the state variable "Costs of Introducing a WfMS". The "Reduction Rate" controls its decrease.

A rate is usually determined by other system variables respectively their causal influence. However, systems consist not only of state variables. There are also variables that

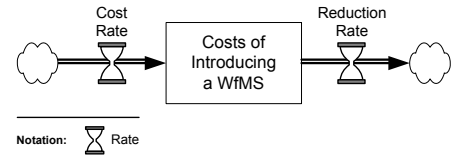

Figure 6. Controlling Inflows and Outflows.

are not subject to accumulation. These variables are called auxiliary variables. Regarding the determination of rates, both state and auxiliary variables can influence a rate. As example consider Figure 7.

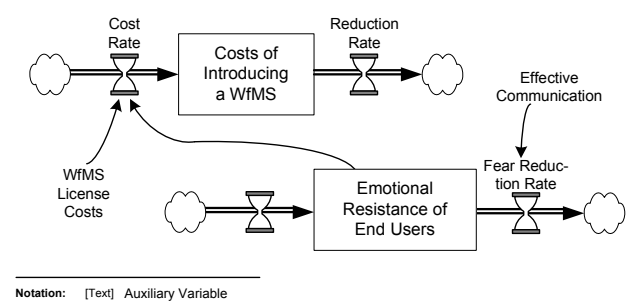

Figure 7. Controlling Rates.

The "Cost Rate" is influenced by an auxiliary variable "WfMS License Costs". The same rate is also influenced by the state variable "Emotional Resistance of End Users". The latter is reasonable as the costs for introducing a WfMS are significantly influenced by user resistance $[12,20]$. Emotional resistance, in turn, can be decreased by an early and effective communication of background information regarding the introduction of a WfMS.

The correct implementation and validity of evaluation 
models is typically ensured by tests. Forrester [8], for example, describes 17 tests that can be used to verify and validate a model's structure and behavior. Richardson and Pugh [19] recommend using test functions, to conduct hypothesis tests, and to do sensitivity analysis.

\subsection{Model Interpretation}

The fourth step of our evaluation methodology deals with the interpretation of evaluation models (i.e., with the derivation of conclusions). In order to understand how a given system "works" and what policies might change its behavior, we use computer simulation.

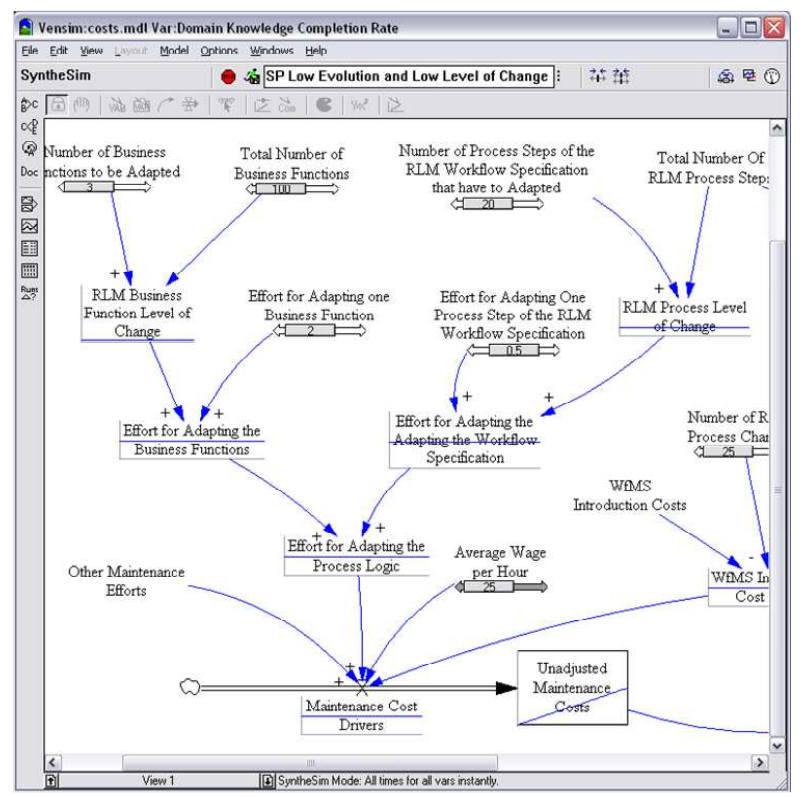

Figure 8. Using Vensim [2] for Simulation.

The use of simulation becomes necessary as systems of variables tend to get complex. This complexity often misguides our intuition about the system's behavior (i.e., about the interactions of the variables). Simulation, by contrast, enables us to trace selected variables along a defined time horizon (e.g., along the course of project). However, this does not necessarily imply the exact prediction of a specific model variable based on a given set of initial conditions.

We use the visual modeling tool Vensim [2] to construct, simulate, and analyze our evaluation models (cf. Fig. 8). This tool enables us to accomplish behavioral "experiments" by temporarily changing selected model variables in a series of simulation runs. The values of all model variables are stored for each simulation run. These data can be further analyzed with different graphical chart tools.

Typically, at least two simulation runs are performed. A first run (assuming a variable of interest as "low") defines the baseline for further comparisons. A second run depicts the effects when the variable of interest is changed. The results of several simulation runs are finally compared (based on graphical charts) to derive conclusions.

\section{Related Work}

We discuss related work along the three evaluation perspectives described in Section 2.

Several papers address the impact of WfMS on business process performance (evaluation perspective EP1). Oba et al. [14] analyze the introduction of WfMS and particularly focus on the identification of factors that influence work efficiency, processing time, and business process standardization. A mathematical model is provided for predicting the reduction rate of processing times. An extension is the work of Reijers and van der Aalst [18]. They use process simulation to compare pre- and post-implementations of information systems that rely on workflow management technology. Their focus is on analyzing business process performance based on criteria such as lead time, waiting time, service time, and utilization of resources. The use of workflow management technology has resulted in most cases in a significant decrease of lead and service time. Choenni et al. [7] present a model to measure the added value of WfMS to business processes that builds upon different performance criteria such as speed, quality, flexibility, and reliability. A performance criterion is a parameter of a business process that is improved or compounded by the introduction of a WfMS. The overall economic impact of a WfMS is calculated from the costs related to these four performance criteria. Aiello [4] introduces a measurement framework for the evaluation of workflows. The framework is defined in an abstract setting to enable generality and ensure independence from existing WfMS.

Only few approaches deal with the economic impact of WfMS on software development and software maintenance (evaluation perspectives EP2 and EP3). Parkes, for example, analyzes critical success factors for WfMS implementations based on a survey [16] and a case study [17]. Three critical success factors are considered as being of particular importance: management commitment, communication, and participation by end users. Empirical studies [11] also indicate that the effort for realizing process-oriented applications can be significantly reduced when using WfMS.

There are other approaches that deal with further aspects regarding the economics of WfMS. Becker et. al [6] have developed a framework to identify those processes that can be supported by WfMS in a "profitable" way. Their framework can serve as guideline for evaluating processes during the selection and introduction of a WfMS. It contains three groups of criteria: technical, organizational and eco- 
nomic. Designed as a scoring model, their approach enables users to systematically determine those business processes that can be automated using a WfMS. A different approach is proposed by Abate et al. [3], who introduce a novel measurement language to evaluate the performance of automated business processes: the "workflow performance query language" (WPQL). This language allows to define and to perform measurements independent from a specific WfMS implementation. It provides different mechanisms to select the workflow entities that are to be measured. Constructs for defining metrics are also defined.

All these approaches analyze very specific facets (e.g., business process performance improvements). However, the combined incorporation of all three evaluation perspectives is addressed by none of them. Our approach, by contrast, enables practitioners as well as researchers to evaluate WfMS from a holistic perspective as all three evaluation perspectives can be addressed.

\section{Summary and Outlook}

This paper presents a methodology for evaluating costs and benefits that arise when introducing WfMS. Doing so, we take a value-based perspective. In particular, our approach allows for the analysis of WfMS economics based on the design and simulation of evaluation models. These models follow the System Dynamics notation. Cost and impact factors as well as their dependencies are considered as "economic systems" whose structure and behavior are modeled and simulated to make economic effects transparent.

Next steps will include the design and analysis of more complex evaluation models. This implies the partial empirical validation of underlying assumptions based on expert interviews, controlled experiments, and case studies. Besides, it is our goal to identify further impact factors (and suitable metrics to quantify them). Finally, we also want to deliberate whether we can use our approach for the economic-driven evaluation of other process-oriented software technologies [13] as well (e.g., business process integration technologies).

\section{Acknowledgement}

The authors would like to thank Stefanie Rinderle (University of Ulm) for her valuable contribution to this paper.

\section{References}

[1] Economic-driven Evaluations of Process-oriented Software Technologies (EcoPOST). Project Homepage: www.mutschler.info/ecopost, 2006.

[2] Ventana Systems. Vensim: http://www.vensim.com/, 2006.
[3] A. F. Abate, A. Esposito, N. Grieco, and G. Nota. Workflow Performance Evaluation through WPQL. Proc. 14th Int'l. Conf. Software Engineering and Knowledge Engineering (SEKE '02), pp.489-495, 2002.

[4] R. Aiello. Workflow Performance Evaluation. PhD Thesis, University of Salerno, Italy, 2004.

[5] Y. L. Antonucci. Using Workflow Technologies to improve Organizational Competitiveness. Int'1. Journal of Management, 14(1), pp.117-126, 1997.

[6] J. Becker, C. v. Uthmann, M. zur Muehlen, and M. Rosemann. Identifying the Workflow Potential of Business Processes. Proc. 32nd Hawaii Int'l. Conf. on System Sciences (HICSS '99), 1999.

[7] S. Choenni, R. Bakkera, and W. Baetsa. On the Evaluation of Workflow Systems in Business Processes. Electronic Journal of Information Systems Evaluation (EJISE), 6(2), 2003.

[8] J. Forrester and P. Senge. Tests for Building Confidence in $S$ - $D$ Models. TIMS Studies in the Management Sciences, volume 14, pp.208-228, 1980.

[9] J. W. Forrester. Industrial Dynamics. Productivity Press, Cambridge, London, 1961.

[10] T. Haeberlein. Understanding Software Acquisition through Modeling and Simulation. PhD Thesis, University of Ulm, Germany, 2005.

[11] N. Kleiner. Can Business Process Changes Be Cheaper Implemented with Workflow-Management-Systems? Proc. Int'l. Conf. Information Resources Management Association, pp.529-532, 2004.

[12] P. Kueng. The Effects of Workflow Systems on Organization: A Qualitative Study. Proc. Business Process Management, LNCS 1806, pp. 301-316, 2000.

[13] B. Mutschler, M. Reichert, and J. Bumiller. Designing an Economic-driven Evaluation Framework for Processoriented Software Technologies. Proc. 28th Int'1. Conf. on Software Engineering (ICSE '06), pp.885-888, Shanghai, China, 2006.

[14] M. Oba, S. Onoda, and N. Komoda. Evaluating the Quantitative Effects of Workflow Systems based on Real Cases. Proc. 33rd Hawaii Int'l. Conf. on System Sciences (HICSS '00), 2000.

[15] K. Ogata. System Dynamics. Prentice Hall, 2003.

[16] A. Parkes. Critical Success Factors in Workflow Implementation. Proc. 6th Pacific Asia Conference on Information Systems (PACIS '02), pp.363-380, 2002.

[17] A. Parkes. A Case Study of Workflow Implementation Success Factors. Proc. 15th Australasian Conf. on Information Systems (ACIS '04), 2004.

[18] H. A. Reijers and W. M. P. van der Aalst. The Effectiveness of Workflow Management Systems - Predictions and Lessons Learned. Int'1. Journal of Information Management, 25(5), pp.457-471, 2005.

[19] G. P. Richardson and A. L. Pugh. System Dynamics - Modeling with DYNAMO. Productivity Press, Portland, 1981.

[20] A. Sarmento and A. Machado. Impact Evaluation of Organisational Changes Enabled by Workflow Systems. Proc. 6th Int'l. Workshop on Groupware, pp.134-137, 2000.

[21] J. D. Sterman. Business Dynamics - Systems Thinking and Modeling for a Complex World. McGraw-Hill, 2000.

[22] W. M. P. van der Aalst and K. van Hee. Workflow Management. MIT Press, 2004. 